\title{
Periodic solutions to the Liénard type equations with phase attractive singularities
}

\author{
Robert Hakl ${ }^{1 *}$ and Manuel Zamora ${ }^{2}$
}

\footnotetext{
"Correspondence: hakl@ipm.cz ${ }^{1}$ Institute of Mathematics, Academy of Sciences of the Czech Republic, Žižkova 22, Brno, 616 62, Czech Republic

Full list of author information is available at the end of the article
}

\begin{abstract}
Sufficient conditions are established guaranteeing the existence of a positive $\omega$-periodic solution to the equation

$$
u^{\prime \prime}+f(u) u^{\prime}+g(u)=h(t, u)
$$

where $f, g:(0,+\infty) \rightarrow \mathbb{R}$ are continuous functions with possible singularities at zero and $h:[0, \omega] \times \mathbb{R} \rightarrow \mathbb{R}$ is a Carathéodory function. The results obtained are rewritten for the equation of the type

$$
u^{\prime \prime}+\frac{c u^{\prime}}{u^{\mu}}+\frac{g_{1}}{u^{\nu}}-\frac{g_{2}}{u^{\gamma}}=h_{0}(t) u^{\delta}
$$

where $g_{1}, g_{2}, \delta$ are non-negative constants, $c, \mu, \nu, \gamma$ are real numbers, and $h_{0} \in L([0, \omega] ; \mathbb{R})$. The last equation also covers the so-called Rayleigh-Plesset equation, frequently used in fluid mechanics to model the bubble dynamics in liquid. In the paper, the case when $v>\gamma$, i.e., the case which covers the attractive singularity of the function $g$, is studied. The results obtained assure that there exists a positive $\omega$-periodic solution to the above-mentioned equation if the power $\mu$ or $v$ is sufficiently large.
\end{abstract}

MSC: $34 C 25 ; 34 \mathrm{~B} 16 ; 34 \mathrm{~B} 18 ; 76 \mathrm{~N} 15$

Keywords: Rayleigh-Plesset equation; singular equation; periodic solution; upper and lower function

\section{Introduction}

The topic of singular boundary value problems has been of substantial and rapidly growing interest for many scientists and engineers. The importance of such investigation is emphasized by the fact that numerical simulations of solutions to such problems usually break down near singular points.

On the other hand, problems of this type arise frequently in applied science. Namely, in fluid mechanics, since 1917 the physicists have used the Rayleigh equation,

$$
\rho\left[R \ddot{R}+\frac{3}{2} \dot{R}^{2}\right]=p(R)-p_{\infty}
$$

to model the bubble dynamics in liquid, where $R(t)$ is the ratio of the bubble at the time $t$, $\rho$ is the liquid density, $p_{\infty}$ is the pressure in the liquid at a large distance from the bubble,

\section{黛 Springer}

(c) 2013 Hakl and Zamora; licensee Springer. This is an Open Access article distributed under the terms of the Creative Commons Attribution License (http://creativecommons.org/licenses/by/2.0), which permits unrestricted use, distribution, and reproduction in any medium, provided the original work is properly cited. 
and $p(R)$ is the pressure in the liquid at the bubble boundary. In 1949, Plesset proposed to use a more exact equation involving the surface-tension constant $S$ and the coefficient of the liquid viscosity $\mu_{n}$, which was finally improved by adding a term with polytropic coefficient $k$ in 1977, nowadays known as a Rayleigh-Plesset equation (see [1])

$$
\rho\left[R \ddot{R}+\frac{3}{2} \dot{R}^{2}\right]=\left[P_{\nu}-p_{\infty}(t)\right]+P_{g_{0}}\left(\frac{R_{0}}{R}\right)^{3 k}-\frac{2 S}{R}-\frac{4 \mu \approx \dot{R}}{R} .
$$

The transformation $R=u^{\frac{2}{5}}$ in the previous equation leads to the equation

$$
\ddot{u}=\frac{5\left[P_{v}-p_{\infty}(t)\right]}{2 \rho} u^{\frac{1}{5}}+\left(\frac{5 P_{g_{0}} R_{0}^{3 k}}{2 \rho}\right) \frac{1}{u^{\frac{6 k-1}{5}}}-\frac{5 S}{u^{\frac{1}{5}}}-4 \mu * \frac{\dot{u}}{u^{\frac{4}{5}}} .
$$

Consequently, the class of equations

$$
u^{\prime \prime}(t)+\frac{c u^{\prime}(t)}{u^{\mu}(t)}+\frac{g_{1}}{u^{\nu}(t)}-\frac{g_{2}}{u^{\gamma}(t)}=h_{0}(t) u^{\delta}(t) \quad \text { for a.e. } t \in[0, \omega],
$$

with non-negative constants $g_{1}, g_{2}, \delta$, real numbers $c, \mu, v, \gamma$, and $h_{0} \in L([0, \omega] ; \mathbb{R})$, plays an important role in fluid mechanics. Therefore, the equation

$$
u^{\prime \prime}(t)+f(u(t)) u^{\prime}(t)+g(u(t))=h(t, u(t)) \quad \text { for a.e. } t \in[0, \omega]
$$

subjected to the periodic conditions

$$
u(0)=u(\omega), \quad u^{\prime}(0)=u^{\prime}(\omega)
$$

is investigated in the presented paper. Here, $f, g:(0,+\infty) \rightarrow \mathbb{R}$ are continuous, having possible singularities at zero, and $h:[0, \omega] \times \mathbb{R} \rightarrow \mathbb{R}$ is a Carathéodory function, i.e., $h(\cdot, x):[0, \omega] \rightarrow \mathbb{R}$ is measurable for all $x \in \mathbb{R}, h(t, \cdot): \mathbb{R} \rightarrow \mathbb{R}$ is continuous for a.e. $t \in[0, \omega]$, and for every $r>0$, there exists a non-negative function $q_{r} \in L([0, \omega] ; \mathbb{R})$ such that $|h(t, x)| \leq q_{r}(t)$ for a.e. $t \in[0, \omega]$ and all $|x| \leq r$. By a solution to (1.2), (1.3) we understand a function $u:[0, \omega] \rightarrow \mathbb{R}$ which is positive, absolutely continuous together with its first derivative, satisfies (1.2) almost everywhere on [0, $\omega]$, and verifies (1.3). In spite of the fact that the problem (1.2), (1.3) was investigated by many mathematicians (see, e.g., [2-27]), most of the mentioned works deal with the repulsive case and/or when $f$ has no singularity. However, the physical model, covered by equation (1.1), justifies considering the types of equations with a singular friction-like term.

A particular case of (1.1) is the equation

$$
u^{\prime \prime}+\frac{1}{u^{v}}=h_{0}(t)
$$

studied by Lazer and Solimini. Their results were published in 1987 (see [11]) and they proved, among others, that (1.4), (1.3) has at least one solution if and only if $\bar{h}_{0}>0$, provided $h_{0}$ is bounded. Recently, we have proved (see [28]) that this result cannot be extended to the case when $h_{0}$ is a general integrable (and so unbounded) function unless some additional conditions are introduced. In particular, (1.4), (1.3) is solvable for any 
$h_{0} \in L([0, \omega] ; \mathbb{R})$ with $\bar{h}_{0}>0$ if $v \geq 1$; and, moreover, for any $v \in(0,1)$, there exists a function $h_{0} \in L([0, \omega] ; \mathbb{R})$ with $\bar{h}_{0}>0$ such that (1.4), (1.3) has no solution. At this point, we would like to emphasize the important fact that the condition $v \geq 1$ can be weakened if (1.4) is generalized to equation (1.1), see Remark 2.2 below.

The structure of the paper is as follows. After the introduction and basic notation, we recall the definition of lower and upper functions to the problem (1.2), (1.3), and we formulate the classical theorem on the existence of a solution to (1.2), (1.3) in the case when there exists a couple of well-ordered lower and upper functions. In Section 2, we establish our main results and their consequences. Sections 3 and 4 are devoted to auxiliary propositions and proofs of the main results, respectively.

For convenience, we finish the introduction with a list of notations which are used throughout the paper:

$\mathbb{N}$ is the set of all natural numbers, $\mathbb{R}$ is the set of all real numbers, $\mathbb{R}^{+}=(0,+\infty),[x]_{+}=$ $\max \{x, 0\},[x]_{-}=\max \{-x, 0\}$.

$C([0, \omega] ; \mathbb{R})$ is the Banach space of continuous functions $u:[0, \omega] \rightarrow \mathbb{R}$ with the norm

$$
\|u\|_{\infty}=\max \{|u(t)|: t \in[0, \omega]\} .
$$

$C\left(\mathbb{R}^{+} ; \mathbb{R}\right)$, resp. $C\left(\mathbb{R}^{+} ; \mathbb{R}^{+}\right)$, is the set of continuous functions $u: \mathbb{R}^{+} \rightarrow \mathbb{R}$, resp. $u: \mathbb{R}^{+} \rightarrow$ $\mathbb{R}^{+}$.

$C^{1}\left(\mathbb{R}^{+} ; \mathbb{R}^{+}\right)$is the set of functions $u: \mathbb{R}^{+} \rightarrow \mathbb{R}^{+}$which are continuous together with their first derivative.

$A C^{1}([0, \omega] ; \mathbb{R})$ is a set of all functions $u:[0, \omega] \rightarrow \mathbb{R}$ such that $u$ and $u^{\prime}$ are absolutely continuous.

$L([0, \omega] ; \mathbb{R})$ is the Banach space of the Lebesgue integrable functions $p:[0, \omega] \rightarrow \mathbb{R}$ endowed with the norm

$$
\|p\|_{1}=\int_{0}^{\omega}|p(s)| d s
$$

For a given $p \in L([0, \omega] ; \mathbb{R})$, its mean value is defined by

$$
\bar{p}=\frac{1}{\omega} \int_{0}^{\omega} p(s) d s .
$$

Given $\varphi, \psi \in L([0, \omega] ; \mathbb{R})$, then

$$
\begin{array}{ll}
\Phi_{+}=\int_{0}^{\omega}[\varphi(s)]_{+} d s, & \Phi_{-}=\int_{0}^{\omega}[\varphi(s)]_{-} d s, \\
\Psi_{+}=\int_{0}^{\omega}[\psi(s)]_{+} d s, & \Psi_{-}=\int_{0}^{\omega}[\psi(s)]_{-} d s .
\end{array}
$$

The following definitions of lower and upper functions are suitable for us. For more general definitions, one can see, e.g., [12, Definition 8.2].

Definition 1.1 A function $\alpha \in A C^{1}([0, \omega] ; \mathbb{R})$ is called a lower function to the problem (1.2), (1.3) if $\alpha(t)>0$ for every $t \in[0, \omega]$ and

$$
\begin{aligned}
& \alpha^{\prime \prime}(t)+f(\alpha(t)) \alpha^{\prime}(t)+g(\alpha(t)) \geq h(t, \alpha(t)) \quad \text { for a.e. } t \in[0, \omega], \\
& \alpha(0)=\alpha(\omega), \quad \alpha^{\prime}(0) \geq \alpha^{\prime}(\omega) .
\end{aligned}
$$


Definition 1.2 A function $\beta \in A C^{1}([0, \omega] ; \mathbb{R})$ is called an upper function to the problem (1.2), (1.3) if $\beta(t)>0$ for every $t \in[0, \omega]$ and

$$
\begin{aligned}
& \beta^{\prime \prime}(t)+f(\beta(t)) \beta^{\prime}(t)+g(\beta(t)) \leq h(t, \beta(t)) \quad \text { for a.e. } t \in[0, \omega], \\
& \beta(0)=\beta(\omega), \quad \beta^{\prime}(0) \leq \beta^{\prime}(\omega) .
\end{aligned}
$$

The following theorem is well known in the theory of differential equations (see, e.g., [12, Theorem 8.12]).

Theorem 1.1 Let $\alpha$ and $\beta$ be lower and upper functions to the problem (1.2), (1.3) such that

$$
\alpha(t) \leq \beta(t) \quad \text { for } t \in[0, \omega] .
$$

Then there exists a solution $u$ to the problem (1.2), (1.3) such that

$$
\alpha(t) \leq u(t) \leq \beta(t) \quad \text { for } t \in[0, \omega] .
$$

\section{Main results}

Theorem 2.1 Let $\rho_{0} \in C^{1}\left(\mathbb{R}^{+} ; \mathbb{R}^{+}\right)$and $\rho_{1} \in C\left(\mathbb{R}^{+} ; \mathbb{R}^{+}\right)$be non-decreasing functions, $h_{0}, h_{1} \in L([0, \omega] ; \mathbb{R})$, and $x_{0}>0$ be such that

$$
h_{1}(t) \rho_{1}(x) \leq h(t, x) \leq h_{0}(t) \rho_{0}(x) \text { for a.e. } t \in[0, \omega], x \geq x_{0},
$$

and let there exist $c_{0}, c_{1} \in \mathbb{R}$ such that

$$
\begin{aligned}
& \frac{g(x)}{\rho_{0}(x)} \leq c_{0}<\bar{h}_{0} \quad \text { for } x \geq x_{0}, \\
& \frac{g(x)}{\rho_{1}(x)} \leq c_{1} \leq \bar{h}_{1} \quad \text { for } x \geq x_{0} .
\end{aligned}
$$

Let, moreover, there exist $\lambda \in[0,1]$ such that

$$
\begin{aligned}
& \int_{0}^{1} \frac{d s}{\rho_{0}^{\lambda}(s)}<+\infty, \\
& \lim _{x \rightarrow 0_{+}} \frac{g(x)}{\rho_{0}^{\lambda}(x)}=+\infty,
\end{aligned}
$$

and let either

$$
\int_{0}^{1}\left(\frac{[f(s)]_{+}}{\rho_{0}^{\lambda}(s)}+\frac{[g(s)]_{+}}{\rho_{0}^{2 \lambda}(s)}\right) d s=+\infty, \quad \int_{0}^{1} \frac{[f(s)]_{-}}{\rho_{0}^{\lambda}(s)} d s<+\infty
$$

or

$$
\int_{0}^{1}\left(\frac{[f(s)]_{-}}{\rho_{0}^{\lambda}(s)}+\frac{[g(s)]_{+}}{\rho_{0}^{2 \lambda}(s)}\right) d s=+\infty, \quad \int_{0}^{1} \frac{[f(s)]_{+}}{\rho_{0}^{\lambda}(s)} d s<+\infty .
$$

Furthermore, let us suppose that $\rho_{0}$ fulfills at least one of the following conditions: 
(a) there exists a sequence $\left\{y_{n}\right\}_{n=1}^{+\infty}$ of positive numbers such that

$$
\lim _{n \rightarrow+\infty} y_{n}=+\infty, \quad \lim _{n \rightarrow+\infty} \frac{\rho_{0}^{1-\lambda}\left(y_{n}\right)}{\sigma\left(y_{n}\right)}=0,
$$

and there exist $\varepsilon_{0}>0, \varepsilon_{1} \in\left(0, \varepsilon_{0}\right]$, and $n_{0} \in \mathbb{N}$ such that

$$
\begin{array}{ll}
\frac{\rho_{0}^{1-\lambda}\left(\left(1+\varepsilon_{0}\right) y_{n}\right)}{\rho_{0}^{1-\lambda}\left(y_{n}\right)} \Phi_{-} \leq \Phi_{+}-\varepsilon_{0} & \text { for } n \geq n_{0}, \\
\left(1+\varepsilon_{1}\right) \sigma\left(y_{n}\right) \leq \sigma\left(\left(1+\varepsilon_{0}\right) y_{n}\right) & \text { for } n \geq n_{0},
\end{array}
$$

where $\varphi(t)=h_{0}(t)-c_{0}$ for almost every $t \in[0, \omega]$ and

$$
\sigma(x)=\int_{0}^{x} \frac{d s}{\rho_{0}^{\lambda}(s)}
$$

(b) the function $\frac{\rho_{0}^{1-\lambda}(x)}{\sigma(x)}$ is non-increasing and

$$
\frac{\omega}{4} \Phi_{+} \Phi_{-} \frac{\rho_{0}^{1-\lambda}\left(x_{0}\right)}{\sigma\left(x_{0}\right)}<\Phi_{+}-\Phi_{-}
$$

where $\varphi(t)=h_{0}(t)-c_{0}$ for almost every $t \in[0, \omega]$ and $\sigma$ is given by (2.11). Besides, let us suppose that $\rho_{1}$ fulfills at least one of the following conditions:

(c) there exists a sequence $\left\{z_{n}\right\}_{n=1}^{+\infty}$ of positive numbers such that

$$
\lim _{n \rightarrow+\infty} z_{n}=+\infty, \quad \lim _{n \rightarrow+\infty} \frac{\rho_{1}\left(z_{n}\right)}{z_{n}}=0,
$$

and there exist $\varepsilon_{2}>0$ and $n_{1} \in \mathbb{N}$ such that

$$
\frac{\rho_{1}\left(z_{n}\left(1+\varepsilon_{2}\right)\right)}{\rho_{1}\left(z_{n}\right)} \Psi_{-} \leq \Psi_{+} \quad \text { for } n \geq n_{1}
$$

where $\psi(t)=h_{1}(t)-c_{1}$ for almost every $t \in[0, \omega]$;

(d) the function $\frac{\rho_{1}(x)}{x}$ is non-increasing and

$$
\frac{\omega}{4} \Psi_{+} \Psi_{-} \frac{\rho_{1}\left(x_{0}\right)}{x_{0}} \leq \Psi_{+}-\Psi_{-},
$$

where $\psi(t)=h_{1}(t)-c_{1}$ for almost every $t \in[0, \omega]$.

Then there exists at least one solution to the problem (1.2), (1.3).

Remark 2.1 Note that there exists a suitable $\varepsilon_{1}$ such that (2.10) holds, e.g., if

$$
\limsup _{x \rightarrow+\infty} \frac{\rho_{0}^{\lambda}\left(\left(1+\varepsilon_{0}\right) x\right)}{\rho_{0}^{\lambda}(x)}<1+\varepsilon_{0} .
$$

For equation (1.1), from Theorem 2.1 we get the following assertion. 
Corollary 2.1 Let $g_{1}>0, g_{2} \geq 0,0 \leq \delta<1, v>\gamma, v+\delta>0$ and

$$
\text { either }(\mu+\delta) \operatorname{sgn}|c| \geq 1 \text { or } \quad v+2 \delta \geq 1 .
$$

If

$$
\bar{h}_{0}>-\lim _{x \rightarrow+\infty} \frac{g_{2}}{x^{\gamma+\delta}}
$$

then (1.1), (1.3) has at least one solution.

Remark 2.2 In [28], it is proved, among others, that the equation

$$
u^{\prime \prime}+\frac{1}{u^{v}}=h_{0}(t)
$$

with $h_{0} \in L([0, \omega] ; \mathbb{R})$ and $\bar{h}_{0}>0$, has a positive $\omega$-periodic solution if $v \geq 1$. Moreover, there is also an example introduced showing that for any $v \in(0,1)$, there exists $h_{0} \in L([0, \omega] ; \mathbb{R})$ with $\bar{h}_{0}>0$ such that (2.13), (1.3) has no positive solution.

Corollary 2.1 says that if a friction-like term or sub-linear term are added to (2.13), the condition $v \geq 1$ can be weakened. For example,

$$
u^{\prime \prime}+\frac{u^{\prime}}{u^{\mu}}+\frac{1}{u^{v}}=h_{0}(t)
$$

has a positive solution satisfying (1.3) for any $v>0$ if $\mu \geq 1$, provided $\bar{h}_{0}>0$. Also, the equation

$$
u^{\prime \prime}+\frac{1}{u^{v}}=h_{0}(t) u^{\delta}
$$

subjected to the boundary conditions $(1.3)$ is solvable for any $v>0$ if $\delta \in[1 / 2,1)$, provided $\bar{h}_{0}>0$.

Example 2.1 As it was mentioned in the introduction, the particular case of (1.1) is the so-called Rayleigh-Plesset equation frequently used in fluid mechanics. This equation has the following form:

$$
u^{\prime \prime}+\frac{c u^{\prime}}{u^{\frac{4}{5}}}+\frac{g_{1}}{u^{\frac{1}{5}}}-\frac{g_{2}}{u^{\gamma}}=h_{0}(t) u^{\frac{1}{5}}
$$

where $h_{0} \in L([0, \omega] ; \mathbb{R}), c, g_{1}, g_{2}$ are positive constants and $\gamma \in \mathbb{R}($ see $[9,10])$.

The results dealing with the existence of positive $\omega$-periodic solutions of (2.14) were established in [10] provided $h_{0}$ is bounded from above (see [10, Theorems 4.4, 4.6, 4.7]). However, Corollary 2.1 says that in the case when $\gamma<1 / 5$, the problem $(2.14),(1.3)$ is solvable if one of the following items is fulfilled:

1. $\gamma>-1 / 5$ and $\bar{h}_{0}>0$;

2. $\gamma=-1 / 5$ and $\bar{h}_{0}>-g_{2}$;

3. $\gamma<-1 / 5$.

Thus, Corollary 2.1 assures that the boundedness of $h_{0}$ is not necessary. 
Corollary 2.2 Let $g_{1}>0, g_{2} \geq 0, v>\gamma, v+1>0$. Let, moreover, either $g^{*}=-\infty$ or

$$
\bar{h}_{0}>g^{*}>-\infty, \quad \frac{\omega}{4} \Phi_{+} \Phi_{-}<\Phi_{+}-\Phi_{-}
$$

where $\varphi(t)=h_{0}(t)-g^{*}$ for almost every $t \in[0, \omega]$, and

$$
g^{*}=-\lim _{x \rightarrow+\infty} \frac{g_{2}}{x^{\gamma+1}}
$$

Then the problem (1.1), (1.3) with $\delta=1$ has at least one solution.

Remark 2.3 According to [29] and Theorem 1.1, it can be easily verified that the problem

$$
u^{\prime \prime}+\frac{g_{1}}{u^{v}}=h_{0}(t) u ; \quad u(0)=u(\omega), \quad u^{\prime}(0)=u^{\prime}(\omega)
$$

with $g_{1}>0$ and $v>0$, has a positive solution if and only if the inclusion $\mathcal{L}\left[0,-h_{0}\right] \in V^{-}$ holds (see notation in [29]).

Indeed, according to [29, Definition 1.1], the inclusion $\mathcal{L}\left[0,-h_{0}\right] \in V^{-}$implies the existence of a positive solution $v$ to the problem

$$
v^{\prime \prime}=h_{0}(t) v-g_{1} ; \quad u(0)=u(\omega), \quad u^{\prime}(0)=u^{\prime}(\omega) .
$$

Therefore there exist constants $x>0$ and $y>0$ such that $x^{1+v} \leq v(t) \leq y^{1+v}$ for $t \in[0, \omega]$. By setting

$$
\alpha(t) \stackrel{\text { def }}{=} \frac{v(t)}{y^{v}}, \quad \beta(t) \stackrel{\text { def }}{=} \frac{v(t)}{x^{v}} \quad \text { for } t \in[0, \omega],
$$

one can easily realize that $\alpha$ and $\beta$ are, respectively, lower and upper functions to (2.16) satisfying (1.5). Now, the existence of a positive solution to (2.16) follows from Theorem 1.1.

On the other hand, the existence of a positive solution to (2.16) implies the inclusion $\mathcal{L}\left[0,-h_{0}\right] \in V^{-}$(see $[29$, Theorem 2.1]).

However, one of the optimal effective conditions guaranteeing such an inclusion is $h_{0} \not \equiv$ 0 and

$$
\frac{\omega}{4} \int_{0}^{\omega}\left[h_{0}(s)\right]_{+} d s \int_{0}^{\omega}\left[h_{0}(s)\right]_{-} d s \leq \int_{0}^{\omega}\left[h_{0}(s)\right]_{+} d s-\int_{0}^{\omega}\left[h_{0}(s)\right]_{-} d s
$$

(see [29, Corollary 2.5]). Therefore, the condition (2.15) is natural in a certain sense.

When the right-hand side of equation (1.3) does not depend on $u$, i.e., when $h(t, x) \equiv$ $h_{0}(t)$, then (1.3) has the form

$$
u^{\prime \prime}(t)+f(u(t)) u^{\prime}(t)+g(u(t))=h_{0}(t) \quad \text { for a.e. } t \in[0, \omega]
$$

From Theorem 2.1, for equation (2.17) we get the following assertion.

Corollary 2.3 Let there exist $x_{0}>0$ and $c_{0} \in \mathbb{R}$ such that

$$
g(x) \leq c_{0}<\bar{h}_{0} \quad \text { for } x \geq x_{0}
$$


and let

$$
\lim _{x \rightarrow 0_{+}} g(x)=+\infty
$$

Let, moreover, either

$$
\int_{0}^{1}\left([f(s)]_{+}+[g(s)]_{+}\right) d s=+\infty, \quad \int_{0}^{1}[f(s)]_{-} d s<+\infty
$$

or

$$
\int_{0}^{1}\left([f(s)]_{-}+[g(s)]_{+}\right) d s=+\infty, \quad \int_{0}^{1}[f(s)]_{+} d s<+\infty .
$$

Then there exists at least one solution to the problem (2.17), (1.3).

In the following result, the assumptions do not depend on the friction-like term. On the other hand, a certain smallness of oscillation of the primitive to $h_{0}$ is supposed. Clearly, Theorems 2.1 and 2.2 are independent.

Theorem 2.2 Let $\rho_{0} \in C^{1}\left(\mathbb{R}^{+} ; \mathbb{R}^{+}\right)$and $\rho_{1} \in C\left(\mathbb{R}^{+} ; \mathbb{R}^{+}\right)$be non-decreasing functions, $h_{0}, h_{1} \in L([0, \omega] ; \mathbb{R})$, and $0<x_{0} \leq x_{1}<+\infty$ be such that

$$
\begin{aligned}
& h(t, x) \leq h_{0}(t) \rho_{0}(x) \quad \text { for a.e. } t \in[0, \omega], 0<x \leq x_{0}, \\
& h(t, x) \geq h_{1}(t) \rho_{1}(x) \quad \text { for a.e. } t \in[0, \omega], x \geq x_{1} .
\end{aligned}
$$

Let, moreover,

$$
\begin{aligned}
& \frac{\omega}{8}\left\|h_{0}-\bar{h}_{0}\right\|_{1}<\int_{0}^{x_{0}} \frac{d s}{\rho_{0}(s)}<+\infty, \\
& \frac{g(x)}{\rho_{0}(x)} \geq \bar{h}_{0} \quad \text { for } 0<x \leq x_{0},
\end{aligned}
$$

and let there exist $c_{1} \in \mathbb{R}$ such that

$$
\frac{g(x)}{\rho_{1}(x)} \leq c_{1} \leq \bar{h}_{1} \quad \text { for } x \geq x_{1} .
$$

Besides, let us suppose that $\rho_{1}$ fulfills at least one of the conditions (c) or (d) of Theorem 2.1. Then there exists at least one solution to the problem (1.2), (1.3).

In the particular case, when equation (1.2) has the form (1.1), the following assertion immediately follows from Theorem 2.2 .

Corollary 2.4 Let $0 \leq \delta<1$, and let $0<x_{0} \leq x_{1}<+\infty$ be such that

$$
(1-\delta) \frac{\omega}{8}\left\|h_{0}-\bar{h}_{0}\right\|_{1}<x_{0}^{1-\delta}
$$


Let, moreover,

$$
\begin{aligned}
& \frac{g_{1}}{x^{\nu+\delta}}-\frac{g_{2}}{x^{\gamma+\delta}} \geq \bar{h}_{0} \quad \text { if } 0<x \leq x_{0}, \\
& \frac{g_{1}}{x^{\nu+\delta}}-\frac{g_{2}}{x^{\gamma+\delta}} \leq \bar{h}_{0} \quad \text { if } x \geq x_{1} .
\end{aligned}
$$

Then the problem (1.1), (1.3) has at least one solution.

Remark 2.4 The consequence of Theorem 2.2 for the problem (2.17), (1.3) coincides with the result obtained in [10, Theorem 3.6].

\section{Auxiliary propositions}

In what follows, we will show the existence of a solution to the equation

$$
u^{\prime \prime}(t)+f(u(t)) u^{\prime}(t)+g(u(t))=h_{0}(t) \rho_{0}(u(t)) \text { for a.e. } t \in[0, \omega]
$$

satisfying the boundary conditions (1.3). Here, $\rho_{0} \in C\left(\mathbb{R}^{+} ; \mathbb{R}^{+}\right)$is a non-decreasing function, $h_{0} \in L([0, \omega] ; \mathbb{R})$, and $f, g \in C\left(\mathbb{R}^{+} ; \mathbb{R}\right)$. Together with (3.1), for every $k \in \mathbb{N}$, consider the auxiliary equation

$$
u^{\prime \prime}(t)+f(u(t)) u^{\prime}(t)+g(u(t))=h_{0 k}(t) \rho_{0}(u(t)) \text { for a.e. } t \in[0, \omega]
$$

where

$$
h_{0 k}(t)=\left\{\begin{array}{ll}
k & \text { if } h_{0}(t)>k, \\
h_{0}(t) & \text { if } h_{0}(t) \leq k
\end{array} \quad \text { for a.e. } t \in[0, \omega], k \in \mathbb{N}\right.
$$

Obviously,

$$
h_{0 k}(t) \leq h_{0 m}(t) \leq h_{0}(t) \text { for a.e. } t \in[0, \omega], k \leq m \text {, }
$$

and

$$
\lim _{k \rightarrow+\infty} \bar{h}_{0 k}=\bar{h}_{0}
$$

The following three results can be found in [10].

Lemma 3.1 (see [10, Corollary 2.17]) Let $x_{0}>0$ and $c \in \mathbb{R}$ be such that

$$
\frac{g(x)}{\rho_{0}(x)} \leq c \leq \bar{h}_{0} \quad \text { for } x \geq x_{0}
$$

Let, moreover, there exist a sequence $\left\{y_{n}\right\}_{n=1}^{+\infty}$ of positive numbers such that

$$
\lim _{n \rightarrow+\infty} y_{n}=+\infty, \quad \lim _{n \rightarrow+\infty} \frac{\rho_{0}\left(y_{n}\right)}{y_{n}}=0,
$$


and let there exist $\varepsilon>0$ and $n_{0} \in \mathbb{N}$ such that

$$
\frac{\rho_{0}\left((1+\varepsilon) y_{n}\right)}{\rho_{0}\left(y_{n}\right)} \Phi_{-} \leq \Phi_{+} \quad \text { for } n \geq n_{0}
$$

where $\varphi(t)=h_{0}(t)-c$ for almost every $t \in[0, \omega]$. Then there exists an upper function $\beta$ to the problem (3.1), (1.3) satisfying

$$
\beta(t) \geq x_{0} \quad \text { for } t \in[0, \omega] .
$$

Lemma 3.2 (see [10, Corollary 2.18]) Let $x_{0}>0$ and $c \in \mathbb{R}$ be such that (3.6) holds. If $\frac{\rho_{0}(x)}{x}$ is a non-increasing function such that

$$
\frac{\omega}{4} \Phi_{+} \Phi_{-} \frac{\rho_{0}\left(x_{0}\right)}{x_{0}} \leq \Phi_{+}-\Phi_{-}
$$

where $\varphi(t)=h_{0}(t)-c$ for almost every $t \in[0, \omega]$, then there exists an upper function $\beta$ to the problem (3.1), (1.3) satisfying (3.8).

Lemma 3.3 (see [10, Corollary 2.11]) Let $x_{0}>\frac{\omega}{8}\left\|h_{0}-\bar{h}_{0}\right\|_{1}$ be such that

$$
g(x) \geq \bar{h}_{0} \quad \text { for } 0<x \leq x_{0} .
$$

Then there exists a lower function $\alpha$ to the problem (2.17), (1.3) with

$$
0<\alpha(t) \leq x_{0} \quad \text { for } t \in[0, \omega] .
$$

Lemma 3.4 Let $x_{0}>0$ and $c_{0} \in \mathbb{R}$ be such that (2.2) holds. Let, moreover, there exist a sequence $\left\{y_{n}\right\}_{n=1}^{+\infty}$ of positive numbers such that (3.7) is fulfilled, and let there exist $\varepsilon>0$ and $n_{0} \in \mathbb{N}$ such that

$$
\frac{\rho_{0}\left((1+\varepsilon) y_{n}\right)}{\rho_{0}\left(y_{n}\right)} \Phi_{-} \leq \Phi_{+}-\varepsilon \quad \text { for } n \geq n_{0},
$$

where $\varphi(t)=h_{0}(t)-c_{0}$ for almost every $t \in[0, \omega]$. Then there exist $k_{0} \in \mathbb{N}$ and an upper function $\beta$ to the problems (3.2), (1.3) for $k \geq k_{0}$ satisfying (3.8).

Proof Put

$$
\begin{aligned}
& \varphi_{k}(t)=h_{0 k}(t)-c_{0} \quad \text { for a.e. } t \in[0, \omega], \\
& \Phi_{k+}=\int_{0}^{\omega}\left[\varphi_{k}(s)\right]_{+} d s, \quad \Phi_{k-}=\int_{0}^{\omega}\left[\varphi_{k}(s)\right]_{-} d s .
\end{aligned}
$$

Then, obviously, in view of (3.3), we have

$$
\lim _{k \rightarrow+\infty} \Phi_{k+}=\Phi_{+}, \quad \lim _{k \rightarrow+\infty} \Phi_{k-}=\Phi_{-}
$$


and, consequently, on account of (2.2), (3.5), (3.10), and (3.13), there exists $k_{0} \in \mathbb{N}$ such that

$$
\begin{aligned}
& \frac{g(x)}{\rho_{0}(x)} \leq c_{0} \leq \bar{h}_{0 k_{0}} \leq \bar{h}_{0} \quad \text { for } x \geq x_{0}, \\
& \frac{\rho_{0}\left((1+\varepsilon) y_{n}\right)}{\rho_{0}\left(y_{n}\right)} \Phi_{k_{0}-} \leq \Phi_{k_{0}+} \text { for } n \geq n_{0} .
\end{aligned}
$$

Therefore, according to Lemma 3.1, there exists an upper function $\beta$ to (3.2), (1.3) with $k=k_{0}$ satisfying (3.8). Obviously, in view of (3.4) and the non-negativity of $\rho_{0}$, it follows that $\beta$ is also an upper function to (3.2), (1.3) for $k \geq k_{0}$.

Lemma 3.5 Let $x_{0}>0$ and $c_{0} \in \mathbb{R}$ be such that (2.2) holds. If $\frac{\rho_{0}(x)}{x}$ is a non-increasing function such that

$$
\frac{\omega}{4} \Phi_{+} \Phi_{-} \frac{\rho_{0}\left(x_{0}\right)}{x_{0}}<\Phi_{+}-\Phi_{-}
$$

where $\varphi(t)=h_{0}(t)-c_{0}$ for almost every $t \in[0, \omega]$, then there exist $k_{0} \in \mathbb{N}$ and an upper function $\beta$ to the problems (3.2), (1.3) for $k \geq k_{0}$ satisfying (3.8).

Proof Define $\varphi_{k}, \Phi_{k+}$, and $\Phi_{k-}$ by (3.11) and (3.12). Then, obviously, in view of (3.3), we have that (3.13) holds and, consequently, on account of (2.2), (3.5), (3.13), and (3.16), there exists $k_{0} \in \mathbb{N}$ such that (3.14) is valid and

$$
\frac{\omega}{4} \Phi_{k_{0}+} \Phi_{k_{0}-} \frac{\rho_{0}\left(x_{0}\right)}{x_{0}} \leq \Phi_{k_{0}+}-\Phi_{k_{0}-}
$$

Therefore, according to Lemma 3.2, there exists an upper function $\beta$ to (3.2), (1.3) with $k=k_{0}$ satisfying (3.8). Obviously, in view of (3.4) and the non-negativity of $\rho_{0}$, it follows that $\beta$ is also an upper function to (3.2), (1.3) for $k \geq k_{0}$.

Lemma 3.6 Let

$$
\liminf _{x \rightarrow 0+} g(x)>-\infty
$$

and let either

$$
\int_{0}^{1}[f(s)]_{+} d s<+\infty
$$

or

$$
\int_{0}^{1}[f(s)]_{-} d s<+\infty
$$

Then, for every $K>0$, there exists a constant $K_{1}>0$ such that for any $k \in \mathbb{N}$ and any positive solution $u$ of (3.2), (1.3) with

$$
\|u\|_{\infty} \leq K,
$$


we have the estimate

$$
\left\|u^{\prime}\right\|_{\infty} \leq K_{1}
$$

Proof Assume that (3.20) is fulfilled. Let $u$ be a positive solution to (3.2), (1.3) satisfying (3.21). Then there exist $t_{0}, t_{1} \in[0, \omega]$ such that

$$
u\left(t_{0}\right)=\min \{u(t): t \in[0, \omega]\}, \quad u\left(t_{1}\right)=\max \{u(t): t \in[0, \omega]\} .
$$

Define the operator $\vartheta$ of $\omega$-periodic prolongation by

$$
\vartheta(v)(t)= \begin{cases}v(t) & \text { if } t \in[0, \omega], \\ v(t-\omega) & \text { if } t \in(\omega, 2 \omega] .\end{cases}
$$

Then, obviously, from (3.2) and (1.3) it follows that

$$
\begin{aligned}
& \vartheta(u)^{\prime \prime}(t)+f(\vartheta(u)(t)) \vartheta(u)^{\prime}(t)+g(\vartheta(u)(t)) \\
& \quad=\vartheta\left(h_{0 k}\right)(t) \rho_{0}(\vartheta(u)(t)) \quad \text { for a.e. } t \in[0,2 \omega] .
\end{aligned}
$$

The integration of (3.25) from $t_{0}$ to $t$, on account of (3.23), yields

$$
\begin{aligned}
\vartheta(u)^{\prime}(t)= & -\int_{t_{0}}^{t} f(\vartheta(u)(s)) \vartheta(u)^{\prime}(s) d s-\int_{t_{0}}^{t} g(\vartheta(u)(s)) d s \\
& +\int_{t_{0}}^{t} \vartheta\left(h_{0 k}\right)(s) \rho_{0}(\vartheta(u)(s)) d s \quad \text { for } t \in\left[t_{0}, t_{0}+\omega\right] .
\end{aligned}
$$

From (3.21), (3.23), and (3.24) it follows that

$$
0<\vartheta(u)\left(t_{0}\right) \leq \vartheta(u)(t) \leq K \quad \text { for } t \in\left[t_{0}, t_{0}+\omega\right] .
$$

Put

$$
\mu=\sup \left\{[g(s)]_{-}: s \in(0, K]\right\} .
$$

According to (3.18), we have

$$
0 \leq \mu<+\infty
$$

Thus, using (3.3), (3.20), (3.21), and (3.27)-(3.29) in (3.26), we arrive at

$$
\vartheta(u)^{\prime}(t) \leq \int_{0}^{K}[f(s)]_{-} d s+\omega \mu+\left\|h_{0}\right\|_{1} \rho_{0}(K) \quad \text { for } t \in\left[t_{0}, t_{0}+\omega\right] .
$$

Put

$$
K_{1}=\int_{0}^{K}[f(s)]_{-} d s+\omega \mu+\left\|h_{0}\right\|_{1} \rho_{0}(K) .
$$


Then, on account of (3.24) and (3.30), we have

$$
u^{\prime}(t) \leq K_{1} \quad \text { for } t \in[0, \omega]
$$

On the other hand, the integration of (3.25) from $t$ to $t_{1}+\omega$, with respect to (3.23), results in

$$
\begin{aligned}
\vartheta(u)^{\prime}(t)= & \int_{t}^{t_{1}+\omega} f(\vartheta(u)(s)) \vartheta(u)^{\prime}(s) d s+\int_{t}^{t_{1}+\omega} g(\vartheta(u)(s)) d s \\
& -\int_{t}^{t_{1}+\omega} \vartheta\left(h_{0 k}\right)(s) \rho_{0}(\vartheta(u)(s)) d s \quad \text { for } t \in\left[t_{1}, t_{1}+\omega\right] .
\end{aligned}
$$

Now, using (3.3), (3.20), (3.21), and (3.27)-(3.29) in (3.32), we obtain

$$
-\vartheta(u)^{\prime}(t) \leq K_{1} \quad \text { for } t \in\left[t_{1}, t_{1}+\omega\right]
$$

Therefore, in view of (3.24), from (3.33) we get

$$
-u^{\prime}(t) \leq K_{1} \quad \text { for } t \in[0, \omega]
$$

Consequently, (3.31) and (3.34) result in (3.22).

Now, suppose that (3.19) is fulfilled. Put

$$
v(t)=u(\omega-t) \quad \text { for } t \in[0, \omega] .
$$

Then, according to (3.2), we have

$$
v^{\prime \prime}(t)-f(v(t)) v^{\prime}(t)+g(v(t))=\widetilde{h}_{0 k}(t) \rho_{0}(v(t)) \quad \text { for a.e. } t \in[0, \omega]
$$

where

$$
\tilde{h}_{0 k}(t)=h_{0 k}(\omega-t) \quad \text { for a.e. } t \in[0, \omega] \text {. }
$$

Analogously to the above-proved, using (3.19) instead of (3.20), we obtain

$$
\left\|v^{\prime}\right\|_{\infty} \leq K_{1}
$$

with

$$
K_{1}=\int_{0}^{K}[f(s)]_{+} d s+\omega \mu+\left\|h_{0}\right\|_{1} \rho_{0}(K) .
$$

Thus, (3.35) and (3.37) yield (3.22).

Lemma 3.7 Let

$$
\lim _{x \rightarrow 0_{+}} g(x)=+\infty
$$


and let either

$$
\int_{0}^{1}\left([f(s)]_{+}+[g(s)]_{+}\right) d s=+\infty, \quad \int_{0}^{1}[f(s)]_{-} d s<+\infty
$$

or

$$
\int_{0}^{1}\left([f(s)]_{-}+[g(s)]_{+}\right) d s=+\infty, \quad \int_{0}^{1}[f(s)]_{+} d s<+\infty .
$$

Then, for every $K>0$, there exists a constant $a>0$ such that for any $k \in \mathbb{N}$ and any positive solution $u$ of (3.2), (1.3) satisfying (3.21), we have the estimate

$$
a \leq u(t) \quad \text { for } t \in[0, \omega] .
$$

Proof Let $u$ be a positive solution to (3.2), (1.3) satisfying (3.21). Thus, the integration of (3.2) from 0 to $\omega$, in view of (1.3) and (3.4), yields

$$
\int_{0}^{\omega} g(u(s)) d s \leq\left\|h_{0}\right\|_{1} \rho_{0}(K)
$$

On the other hand, (3.38) implies the existence of $x_{0} \in(0,+\infty)$ such that

$$
g(x)>\frac{\left\|h_{0}\right\|_{1} \rho_{0}(K)}{\omega} \geq 0 \quad \text { for } x \in\left(0, x_{0}\right) .
$$

Let $t_{m} \in[0, \omega]$ be such that

$$
u\left(t_{m}\right)=\min \{u(t): t \in[0, \omega]\} .
$$

Obviously, either

$$
u\left(t_{m}\right) \geq x_{0}
$$

or

$$
u\left(t_{m}\right)<x_{0} .
$$

Obviously, it is sufficient to show the estimate (3.41) is valid just in the case when (3.45) is fulfilled. Let, therefore, (3.45) hold.

If $u(t)<x_{0}$ for $t \in[0, \omega]$, then applying (3.43) in (3.42) we obtain a contradiction. Thus, there exist points $t_{1}, t_{2} \in\left(t_{m}, t_{m}+\omega\right)$ such that

$$
\begin{array}{ll}
\vartheta(u)(t)<x_{0} & \text { for } t \in\left[t_{m}, t_{1}\right), \vartheta(u)\left(t_{1}\right)=x_{0}, \\
\vartheta(u)(t)<x_{0} & \text { for } t \in\left(t_{2}, t_{m}+\omega\right], \vartheta(u)\left(t_{2}\right)=x_{0},
\end{array}
$$

where $\vartheta$ is the operator defined by (3.24). Obviously, (3.25) holds. 
Assume that (3.39) holds. Then, according to Lemma 3.6, there exists $K_{1}>0$ such that (3.22) holds. The integration of (3.25) from $t_{m}$ to $t_{1}$, in view of (3.4), (3.21), (3.22), (3.39), (3.43), (3.44), and (3.46), results in

$$
\begin{gathered}
\vartheta(u)^{\prime}\left(t_{1}\right)+\int_{\vartheta(u)\left(t_{m}\right)}^{x_{0}}[f(s)]_{+} d s+\frac{1}{K_{1}} \int_{\vartheta(u)\left(t_{m}\right)}^{x_{0}}[g(s)]_{+} d s \\
\leq \int_{0}^{x_{0}}[f(s)]_{-} d s+\left\|h_{0}\right\|_{1} \rho_{0}(K) .
\end{gathered}
$$

Note that in view of (3.46), we have $\vartheta(u)^{\prime}\left(t_{1}\right) \geq 0$. Consequently, from (3.48) we obtain

$$
\int_{\vartheta(u)\left(t_{m}\right)}^{x_{0}}\left([f(s)]_{+}+[g(s)]_{+}\right) d s \leq K_{2},
$$

where

$$
K_{2}=\left(K_{1}+1\right)\left(\int_{0}^{x_{0}}[f(s)]_{-} d s+\left\|h_{0}\right\|_{1} \rho_{0}(K)\right) .
$$

Note that $K_{2}$ does not depend on $k$. Therefore, if we apply (3.39) in (3.49), it can be easily seen, with respect to (3.44), that there exists a constant $a>0$ such that (3.41) holds.

If (3.40) holds, we integrate (3.25) from $t_{2}$ to $t_{m}+\omega$ and apply similar steps as above, just using (3.47) instead of (3.46). Finally, we arrive at

$$
\int_{\vartheta(u)\left(t_{m}+\omega\right)}^{x_{0}}\left([f(s)]_{-}+[g(s)]_{+}\right) d s \leq K_{2}
$$

with

$$
K_{2}=\left(K_{1}+1\right)\left(\int_{0}^{x_{0}}[f(s)]_{+} d s+\left\|h_{0}\right\|_{1} \rho_{0}(K)\right) .
$$

Therefore, also in this case, there exists a constant $a>0$ such that (3.41) holds.

Lemma 3.8 Let $x_{0}>0$ and $c_{0} \in \mathbb{R}$ be such that (2.2) holds. Let, moreover, (3.38) be fulfilled, and let either (3.39) or (3.40) be valid. Let, in addition, there exist a sequence $\left\{y_{n}\right\}_{n=1}^{+\infty}$ of positive numbers such that (3.7) holds, and let there exist $\varepsilon>0$ and $n_{0} \in \mathbb{N}$ such that (3.10) is fulfilled, where $\varphi(t)=h_{0}(t)-c_{0}$ for almost every $t \in[0, \omega]$. Then there exists a positive solution u to (3.1), (1.3).

Proof According to Lemma 3.4, there exist $k_{0} \in \mathbb{N}$ and an upper function $\beta$ to the problems (3.2), (1.3) for $k \geq k_{0}$ satisfying (3.8). On the other hand, in view of (3.4) and (3.38), there exists $x_{k} \in\left(0, x_{0}\right]$ for $k \geq k_{0}$ such that

$$
g\left(x_{k}\right) \geq h_{0 k}(t) \rho_{0}\left(x_{k}\right) \quad \text { for a.e. } t \in[0, \omega] .
$$

Thus, if we put $\alpha_{k}(t)=x_{k}$ for $t \in[0, \omega]$, according to Theorem 1.1, there exists a solution $u_{k}$ to (3.2), (1.3) for $k \geq k_{0}$ satisfying

$$
0<\alpha_{k}(t) \leq u_{k}(t) \leq \beta(t) \quad \text { for } t \in[0, \omega]
$$


Moreover, according to Lemmas 3.6 and 3.7, in view of (3.50), there exist constants $K>0$, $K_{1}>0$, and $a>0$, not depending on $k$, such that

$$
\begin{aligned}
& \left\|u_{k}\right\|_{\infty} \leq K, \quad\left\|u_{k}^{\prime}\right\|_{\infty} \leq K_{1} \quad \text { for } k \geq k_{0}, \\
& a \leq u_{k}(t) \quad \text { for } t \in[0, \omega], k \geq k_{0}, \\
& \left|u_{k}^{\prime \prime}(t)\right| \leq f_{0} K_{1}+g_{0}+\left|h_{0}(t)\right| \rho_{0}(K) \quad \text { for a.e. } t \in[0, \omega], k \geq k_{0},
\end{aligned}
$$

where

$$
f_{0}=\max \{|f(x)|: x \in[a, K]\}, \quad g_{0}=\max \{|g(x)|: x \in[a, K]\} .
$$

Therefore, according to the Arzelà-Ascoli theorem, there exist $u_{0} \in C([0, \omega] ; \mathbb{R})$ and $v_{0} \in$ $C([0, \omega] ; \mathbb{R})$ such that

$$
\lim _{k \rightarrow+\infty}\left\|u_{k}-u_{0}\right\|_{\infty}=0, \quad \lim _{k \rightarrow+\infty}\left\|u_{k}^{\prime}-v_{0}\right\|_{\infty}=0 .
$$

Moreover, since $u_{k}$ are solutions to (3.2), (1.3), in view of (3.3), (3.52), and (3.54), we have $u_{0} \in A C^{1}([0, \omega] ; \mathbb{R}), u_{0}^{\prime} \equiv v_{0}$, and $u_{0}$ is a positive solution to (3.1), (1.3).

The following assertion can be proved analogously to Lemma 3.8, just Lemma 3.5 is used instead of Lemma 3.4.

Lemma 3.9 Let $x_{0}>0$ and $c_{0} \in \mathbb{R}$ be such that (2.2) holds. Let, moreover, (3.38) be fulfilled, and let either (3.39) or (3.40) be valid. Let, in addition, $\frac{\rho_{0}(x)}{x}$ be a non-increasing function and let (3.16) be fulfilled, where $\varphi(t)=h_{0}(t)-c_{0}$ for almost every $t \in[0, \omega]$. Then there exists a positive solution $u$ to (3.1), (1.3).

Lemma 3.10 Let $\rho_{0} \in C^{1}\left(\mathbb{R}^{+} ; \mathbb{R}^{+}\right)$be non-decreasing, $x_{0}>0$, and $c_{0} \in \mathbb{R}$ be such that (2.2) holds. Let, moreover, there exist $\lambda \in[0,1]$ such that $(2.4)$ and $(2.5)$ are valid, and let either (2.6) or (2.7) be fulfilled. Let, in addition, there exist a sequence $\left\{y_{n}\right\}_{n=1}^{+\infty}$ of positive numbers such that (2.8) holds and let there exist $\varepsilon_{0}>0, \varepsilon_{1} \in\left(0, \varepsilon_{0}\right]$, and $n_{0} \in \mathbb{N}$ such that (2.9) and (2.10) are fulfilled, where $\varphi(t)=h_{0}(t)-c_{0}$ for almost every $t \in[0, \omega]$ and $\sigma$ is given by (2.11). Then there exists a lower function $\alpha$ to the problem (3.1), (1.3).

Proof Because $\rho_{0}$ is a positive function, from (2.4) and (2.11) we obtain that $\sigma$ is a positive increasing function. Therefore, there exists an inverse function $\sigma^{-1}$ to $\sigma$ which is also increasing. Moreover, in view of (2.4) and (2.11), it follows that

$$
\begin{aligned}
& \lim _{x \rightarrow 0_{+}} \sigma(x)=0, \quad \lim _{x \rightarrow 0_{+}} \sigma^{-1}(x)=0, \\
& \lim _{x \rightarrow+\infty} \sigma(x)=+\infty, \quad \lim _{x \rightarrow+\infty} \sigma^{-1}(x)=+\infty .
\end{aligned}
$$

Consider the auxiliary equation

$$
\begin{aligned}
& u^{\prime \prime}(t)+f\left(\sigma^{-1}(u(t))\right) u^{\prime}(t)+\frac{g\left(\sigma^{-1}(u(t))\right)}{\rho_{0}^{\lambda}\left(\sigma^{-1}(u(t))\right)} \\
& =h_{0}(t) \rho_{0}^{1-\lambda}\left(\sigma^{-1}(u(t))\right) \quad \text { for a.e. } t \in[0, \omega] .
\end{aligned}
$$


Put $z=\sigma(x), z_{0}=\sigma\left(x_{0}\right)$. Then from (2.2) we get

$$
\frac{g\left(\sigma^{-1}(z)\right)}{\rho_{0}\left(\sigma^{-1}(z)\right)} \leq c_{0}<\bar{h}_{0} \quad \text { for } z \geq z_{0}
$$

and, in view of (3.55), from (2.5) we have

$$
\lim _{z \rightarrow 0_{+}} \frac{g\left(\sigma^{-1}(z)\right)}{\rho_{0}^{\lambda}\left(\sigma^{-1}(z)\right)}=+\infty
$$

Furthermore, the substitution $r=\sigma(s)$ in (2.6), resp (2.7), with respect to (2.11) yields

$$
\int_{0}^{1}\left(\left[f\left(\sigma^{-1}(r)\right)\right]_{+}+\frac{\left[g\left(\sigma^{-1}(r)\right)\right]_{+}}{\rho_{0}^{\lambda}\left(\sigma^{-1}(r)\right)}\right) d r=+\infty, \quad \int_{0}^{1}\left[f\left(\sigma^{-1}(r)\right)\right]_{-} d r<+\infty
$$

resp.

$$
\int_{0}^{1}\left(\left[f\left(\sigma^{-1}(r)\right)\right]_{-}+\frac{\left[g\left(\sigma^{-1}(r)\right)\right]_{+}}{\rho_{0}^{\lambda}\left(\sigma^{-1}(r)\right)}\right) d r=+\infty, \quad \int_{0}^{1}\left[f\left(\sigma^{-1}(r)\right)\right]_{+} d r<+\infty .
$$

Moreover, put $z_{n}=\sigma\left(y_{n}\right)$ for $n \in \mathbb{N}$. Then from (2.8), in view of (3.55), we get

$$
\lim _{n \rightarrow+\infty} z_{n}=+\infty, \quad \lim _{n \rightarrow+\infty} \frac{\rho_{0}^{1-\lambda}\left(\sigma^{-1}\left(z_{n}\right)\right)}{z_{n}}=0 .
$$

Finally, (2.10) results in

$$
\sigma^{-1}\left(\left(1+\varepsilon_{1}\right) z_{n}\right) \leq\left(1+\varepsilon_{0}\right) y_{n} \quad \text { for } n \geq n_{0},
$$

and so, since $\rho_{0}$ is a non-decreasing function, from (2.9) we obtain

$$
\frac{\rho_{0}^{1-\lambda}\left(\sigma^{-1}\left(\left(1+\varepsilon_{1}\right) z_{n}\right)\right)}{\rho_{0}^{1-\lambda}\left(\sigma^{-1}\left(z_{n}\right)\right)} \Phi_{-} \leq \Phi_{+}-\varepsilon_{1} \quad \text { for } n \geq n_{0}
$$

Therefore, applying Lemma 3.8, according to (3.57)-(3.62), there exists a positive solution $u$ to the problem (3.56), (1.3).

Now, we put $\alpha(t)=\sigma^{-1}(u(t))$ for $t \in[0, \omega]$, i.e., in view of (2.11),

$$
u(t)=\int_{0}^{\alpha(t)} \frac{d s}{\rho_{0}^{\lambda}(s)} \quad \text { for } t \in[0, \omega]
$$

Obviously, $\alpha \in A C^{1}([0, \omega] ; \mathbb{R})$ is a positive function and

$$
\begin{aligned}
& u^{\prime}(t)=\frac{\alpha^{\prime}(t)}{\rho_{0}^{\lambda}(\alpha(t))} \quad \text { for } t \in[0, \omega] \\
& u^{\prime \prime}(t)=\frac{\alpha^{\prime \prime}(t)}{\rho_{0}^{\lambda}(\alpha(t))}-\frac{\lambda \alpha^{\prime 2}(t) \rho_{0}^{\prime}(\alpha(t))}{\rho_{0}^{1+\lambda}(\alpha(t))} \leq \frac{\alpha^{\prime \prime}(t)}{\rho_{0}^{\lambda}(\alpha(t))} \quad \text { for a.e. } t \in[0, \omega] .
\end{aligned}
$$

Thus, it can be easily seen that $\alpha$ is a lower function to the problem (3.1), (1.3).

Analogously to the proof of Lemma 3.10, one can prove the following assertion applying Lemma 3.9 instead of Lemma 3.8. 
Lemma 3.11 Let $\rho_{0} \in C^{1}\left(\mathbb{R}^{+} ; \mathbb{R}^{+}\right)$be non-decreasing, $x_{0}>0$, and $c_{0} \in \mathbb{R}$ be such that (2.2) holds. Let, moreover, there exist $\lambda \in[0,1]$ such that (2.4) and (2.5) are valid, and let either (2.6) or (2.7) be fulfilled. Let, in addition, $\frac{\rho_{0}^{1-\lambda}(x)}{\sigma(x)}$ be a non-increasing function and let (2.12) be fulfilled, where $\varphi(t)=h_{0}(t)-c_{0}$ for almost every $t \in[0, \omega]$ and $\sigma$ is given by (2.11). Then there exists a lower function $\alpha$ to the problem (3.1), (1.3).

\section{Proofs of the main results}

Proof of Theorem 2.1 According to Lemmas 3.1, 3.2, 3.10, and 3.11, the conditions of the theorem guarantee a well-ordered couple of lower and upper functions, therefore the result is a direct consequence of Theorem 1.1.

Proof of Corollary 2.1 It follows from Theorem 2.1 with $h_{1} \equiv h_{0}, \rho_{0}(x)=\rho_{1}(x)=x^{\delta}, \lambda=1$, and $c_{0}=c_{1}$ such that

$$
\bar{h}_{0}>c_{0}>-\lim _{x \rightarrow+\infty} \frac{g_{2}}{x^{\gamma+\delta}} .
$$

Then items (a) and (c) of Theorem 2.1 are fulfilled.

Proof of Corollary 2.2 It follows from Theorem 2.1 with $h_{1} \equiv h_{0}, \rho_{0}(x)=\rho_{1}(x)=x$, and $\lambda<1$ such that $v+\lambda>0, v+2 \lambda \geq 1$. Then items (b) and (d) of Theorem 2.1 are fulfilled.

Proof of Corollary 2.3 It immediately follows from Theorem 2.1 with $h_{1} \equiv h_{0}, \rho_{i}(x) \equiv 1$ $(i=0,1)$.

Proof of Theorem 2.2 Put

$$
\sigma(x)=\int_{0}^{x} \frac{d s}{\rho_{0}(s)} \quad \text { for } x \geq 0 .
$$

Because $\rho_{0}$ is a positive function, from (2.20) and (4.1) we obtain that $\sigma$ is an increasing function. Therefore, there exists an inverse function $\sigma^{-1}$ to $\sigma$ which is also increasing.

Consider the auxiliary equation

$$
u^{\prime \prime}(t)+f\left(\sigma^{-1}(u(t))\right) u^{\prime}(t)+\frac{g\left(\sigma^{-1}(u(t))\right)}{\rho_{0}\left(\sigma^{-1}(u(t))\right)}=h_{0}(t) \quad \text { for a.e. } t \in[0, \omega] .
$$

Put $z=\sigma(x), z_{0}=\sigma\left(x_{0}\right)$. Then from (2.20) and (2.21), in view of (4.1), we get

$$
\begin{aligned}
& \frac{\omega}{8}\left\|h_{0}-\bar{h}_{0}\right\|_{1}<z_{0}, \\
& \frac{g\left(\sigma^{-1}(z)\right)}{\rho_{0}\left(\sigma^{-1}(z)\right)} \geq \bar{h}_{0} \quad \text { for } 0<z \leq z_{0} .
\end{aligned}
$$

Therefore, according to Lemma 3.3, there exists a lower function $w$ to the problem (4.2), (1.3) satisfying

$$
0<w(t) \leq \sigma\left(x_{0}\right) \quad \text { for } t \in[0, \omega]
$$


Now, we put $\alpha=\sigma^{-1}(w(t))$ for $t \in[0, \omega]$, i.e., in view of (4.1),

$$
w(t)=\int_{0}^{\alpha(t)} \frac{d s}{\rho_{0}(s)} \quad \text { for } t \in[0, \omega]
$$

Obviously, with respect to (4.3), $\alpha \in A C^{1}([0, \omega] ; \mathbb{R})$ is a positive function satisfying (3.9), and

$$
\begin{aligned}
& w^{\prime}(t)=\frac{\alpha^{\prime}(t)}{\rho_{0}(\alpha(t))} \quad \text { for } t \in[0, \omega], \\
& w^{\prime \prime}(t)=\frac{\alpha^{\prime \prime}(t)}{\rho_{0}(\alpha(t))}-\frac{\alpha^{\prime 2}(t) \rho_{0}^{\prime}(\alpha(t))}{\rho_{0}^{2}(\alpha(t))} \leq \frac{\alpha^{\prime \prime}(t)}{\rho_{0}(\alpha(t))} \quad \text { for a.e. } t \in[0, \omega] .
\end{aligned}
$$

Thus, on account of (2.18), (3.9), and (4.2), it can be easily seen that $\alpha$ is a lower function to the problem (1.2), (1.3).

The existence of an upper function $\beta$ to (1.2), (1.3) satisfying

$$
\beta(t) \geq x_{1} \quad \text { for } t \in[0, \omega]
$$

follows from (2.19) and Lemma 3.1, resp. 3.2.

Obviously, in view of (3.9) and (4.4), we have that (1.5) holds. Thus the theorem follows from Theorem 1.1.

Proof of Corollary 2.4 It follows from Theorem 2.2 with $h_{1} \equiv h_{0}, \rho_{0}(x)=\rho_{1}(x)=x^{\delta}$, and $c_{1}=\bar{h}_{0}$.

\section{Competing interests}

The authors declare that they have no competing interests.

\section{Authors' contributions}

$\mathrm{RH}$ and $\mathrm{MZ}$ obtained the results in a joint research. Both authors read and approved the final manuscript.

\section{Author details}

${ }^{1}$ Institute of Mathematics, Academy of Sciences of the Czech Republic, Žižkova 22, Brno, 616 62, Czech Republic.

${ }^{2}$ Departamento de Matemática Aplicada, Facultad de Ciencias, Universidad de Granada, Campus de Fuentenueva s/n,

Granada, 18071, Spain.

\section{Acknowledgements}

The first author was supported by RVO: 67985840; the second author was supported by Ministerio de Educación y

Ciencia, Spain, project MTM2011-23652.

Received: 10 December 2012 Accepted: 18 February 2013 Published: 6 March 2013

\section{References}

1. Plesset, MS, Prosperetti, A: Bubble dynamics and cavitation. Annu. Rev. Fluid Mech. 9, 145-185 (1977)

2. Habets, P, Sanchez, L: Periodic solutions of some Liénard equations with singularities. Proc. Am. Math. Soc. 109, 1135-1144 (1990)

3. Bonheure, D, Fabry, C, Smets, D: Periodic solutions of forced isochronous oscillators at resonance. Discrete Contin Dyn. Syst. 8(4), 907-930 (2002)

4. Bonheure, D, De Coster, C: Forced singular oscillators and the method of upper and lower solutions. Topol. Methods Nonlinear Anal. 22, 297-317 (2003)

5. Martins, RF: Existence of periodic solutions for second-order differential equations with singularities and the strong force condition. J. Math. Anal. Appl. 317, 1-13 (2006)

6. Mawhin, J: Topological degree and boundary value problems for nonlinear differential equations. In: Furi, M, Zecca, P (eds.) Topological Methods for Ordinary Differential Equations. Lecture Notes in Mathematics, vol. 1537, pp. 74-142. Springer, Berlin (1993)

7. Omari, $\mathrm{P}, \mathrm{Ye}, \mathrm{W}$ : Necessary and sufficient conditions for the existence of periodic solutions of second-order ordinary differential equations with singular nonlinearities. Differ. Integral Equ. 8, 1843-1858 (1995) 
8. Yuan, R, Zhang, Z: Existence of positive periodic solutions for the Liénard differential equations with weakly repulsive singularity. Acta Appl. Math. 111, 171-178 (2010)

9. Hakl, R, Torres, P, Zamora, M: Periodic solutions of singular second order differential equations: the repulsive case. Topol. Methods Nonlinear Anal. 39, 199-220 (2012)

10. Hakl, R, Torres, P, Zamora, M: Periodic solutions of singular second order differential equations: upper and lower functions. Nonlinear Anal. 74, 7078-7093 (2011)

11. Lazer, AC, Solimini, S: On periodic solutions of nonlinear differential equations with singularities. Proc. Am. Math. Soc. 99, 109-114 (1987)

12. Rachůnková, I, Staněk, S, Tvrdý, M: Solvability of Nonlinear Singular Problems for Ordinary Differential Equations. Contemporary Mathematics and Its Applications, vol. 5, Hindawi Publishing Corporation, New York (2008)

13. Zhang, M: Periodic solutions of Liénard equations with singular forces of repulsive type. J. Math. Anal. Appl. 203, 254-269 (1996)

14. Chu, J, Li, M: Positive periodic solutions of Hill's equation with singular nonlinear perturbations. Nonlinear Anal. 69, 276-286 (2008)

15. Chu, J, Nieto, JJ: Recent existence results for second-order singular periodic differential equations. Bound. Value Probl. 2009, Article ID 540863 (2009)

16. Chu, J, Torres, PJ: Applications of Schauder's fixed point theorem to singular differential equations. Bull. Lond. Math Soc. $39,653-660$ (2007)

17. Chu, J, Torres, PJ, Zhang, M: Periodic solutions of second order non-autonomous singular dynamical systems. J. Differ. Equ. 239, 196-212 (2007)

18. Franco, D, Torres, PJ: Periodic solutions of singular systems without the strong force condition. Proc. Am. Math. Soc. $136,1229-1236(2008)$

19. Franco, D, Webb, JRL: Collisionless orbits of singular and nonsingular dynamical systems. Discrete Contin. Dyn. Syst. $15,747-757(2006)$

20. Hakl, R, Torres, P: On periodic solutions of second-order differential equations with attractive-repulsive singularities. J. Differ. Equ. 248, 111-126 (2010)

21. Rachůnková, I, Tvrdý, M, Vrkoč, I: Existence of nonnegative and nonpositive solutions for second order periodic boundary value problems. J. Differ. Equ. 176, 445-469 (2001)

22. Torres, PJ: Existence of one-signed periodic solutions of some second order differential equations via a Kranoselskii fixed point theorem. J. Differ. Equ. 190, 643-662 (2003)

23. Torres, PJ: Non-collision periodic solutions of forced dynamical systems with weak singularities. Discrete Contin. Dyn. Syst. 11, 693-698 (2004)

24. Torres, PJ: Weak singularities may help periodic solutions to exists. J. Differ. Equ. 232, 277-284 (2007)

25. Torres, PJ: Existence and stability of periodic solutions for second order semilinear differential equations with a singular nonlinearity. Proc. R. Soc. Edinb., Sect. A, Math. 137, 195-201 (2007)

26. Yan, P, Zhang, M: Higher order nonresonance for differential equations with singularities. Math. Methods Appl. Sci. 26 1067-1074 (2003)

27. Zhang, M: Periodic solutions of equations of Emarkov-Pinney type. Adv. Nonlinear Stud. 6, 57-67 (2006)

28. Hakl, R, Zamora, M: On the open problems connected to the results of Lazer and Solimini. Proc. R. Soc. Edinb., Sect. A, Math. (to appear) http://www.math.cas.cz/fichier/preprints/IM_20120709132356_18.pdf

29. Hakl, R, Torres, P: Maximum and antimaximum principles for a second order differential operator with variable coefficients of indefinite sign. Appl. Math. Comput. 217, 7599-7611 (2011)

doi:10.1186/1687-2770-2013-47

Cite this article as: Hakl and Zamora: Periodic solutions to the Liénard type equations with phase attractive

singularities. Boundary Value Problems 2013 2013:47.

\section{Submit your manuscript to a SpringerOpen ${ }^{\ominus}$ journal and benefit from:}

- Convenient online submission

Rigorous peer review

- Immediate publication on acceptance

Open access: articles freely available online

- High visibility within the field

- Retaining the copyright to your article 